\title{
The numerical ranges of unbounded linear operators
}

\section{Béla Bollobás and Stephen E. Eldridge}

Giles and Joseph (Bull. Austral. Math. Soc. 11 (1974), 31-36), proved that the numerical range of an unbounded operator on a Banach space has a certain density property. They showed, in particular, that the numerical range of an unbounded operator on certain Banach spaces is dense in the scalar field. We prove that the numerical range of an unbounded operator on a Banach space is always dense in the scalar field.

This note is a sequel to [1], but for the sake of completeness we repeat the necessary definitions.

Let $X$ be a Banach space over a field $F$, where $F$ is the real or complex field. Denote by $X^{*}$ the dual of $X$ and by $S(X), S\left(X^{*}\right)$ the unit spheres in $X$ and $X^{*}$. For each $x \in S(X)$ let $f_{x} \in S\left(X^{*}\right)$ be such that $f_{x}(x)=1$. Put

$$
\Pi_{8}=\left\{\left(x, f_{x}\right): x \in S(X)\right\} \text {. }
$$

The pair $\left(X, \Pi_{8}\right)$ is a Banach space with a semi-inner-product. The Lumer numerical range of an operator $T$ on $X$ with a given semi-inner-product is defined as

$$
W(T)=\left\{f_{x}(T x): x \in S(X)\right\} \subset \mathrm{F}
$$

The spatial numerical range of $T$ is

$$
V(T)=\left\{f(T x): x \in S(X), f \in S\left(X^{*}\right), f(x)=1\right\} .
$$

Received 3 September 1974. 
Clearly $W(T) \subset V(T)$ and

$$
V(T)=U\{W(T): \text { all semi-inner-products associated with } \chi\} \text {. }
$$

The following theorem extends a result of Giles and Joseph [1]. The proof makes essential use of the ideas in [1].

THEOREM. Let $T$ be an unbounded iinear operator defined on the whole of a Banach space $X$. Then $W(T)$ (and hence also $V(T)$ ) is dense in the scalar field $F(=R$ or $C)$.

Proof. Let $\alpha \in F$ and $\varepsilon>0$. We shall show that the open ball with centre $\alpha$ and radius $\varepsilon$ contains a cluster point of $W(T)$.

$T$ is not bounded so, by the closed graph theorem, there is a sequence $\left\{x_{n}\right\}$ in $X$ such that $x_{n} \rightarrow 0$ and $T x_{n} \rightarrow y \in S(X)$.

If $y+\delta T y \neq 0$ for some $\delta>0$ then put

$$
z_{\delta}=(y+\delta T y) /\|y+\delta T y\| \in S(X) \text {. }
$$

Since the smooth points are dense on the unit sphere of a finite dimensional normed space, we can choose $\delta>0$ so small that $z=z_{\delta}$ satisfies

$$
\|z-y\|<1,\|T z-T y\|<\varepsilon / 2,
$$

and $z$ is a smooth point of the unit sphere of the subspace of $X$ generated by $y$ and $T y$.

Let $f$ be a tangent functional at $z$. Then

$$
|1-f(y)|=|f(z)-f(y)| \leq\|z-y\|<1,
$$

so $f(y) \neq 0$. Let $\beta \in F$ be such that $f(T z)+\beta f(y)=\alpha$.

Put $y_{n}=z+B x_{n}, z_{n}=y_{n} /\left\|y_{n}\right\|$, and let $f_{n}=f_{z_{n}}$ be such that $\left(z_{n}, f_{n}\right) \in \Pi_{s}$

By the Banach-Alaoglu Theorem, the unit ball of $X^{*}$ is weak-* compact. Therefore $\left\{f_{n}\right\}$ has a weak-* cluster point $f_{\beta}$ with $\left\|f_{\beta}\right\| \leq 1$. As $\left\|y_{n}\right\| \rightarrow 1, f_{\beta}$ is also a weak-* cluster point of $\left\{g_{n}\right\}$, where $g_{n}=f_{n} /\left\|y_{n}\right\|$. clearly 


$$
\begin{aligned}
\left|f_{\beta}(z)-1\right| & =\left|f_{\beta}(z)-f_{n}\left(z_{n}\right)\right| \\
& \leq\left|\left(f_{\beta}(z)-g_{n}(z)\right)\right|+\left|g_{n}\left(\beta x_{n}\right)\right| \\
& \leq\left|\left(f_{\beta}-g_{n}\right) z\right|+|\beta|\left\|x_{n}\right\| /\left\|y_{n}\right\| .
\end{aligned}
$$

Consequently $f_{\beta}(z)=I=\left\|f_{\beta}\right\|$, so $f_{\beta}$ is a tangent functional at $z$. Note now that

$$
\begin{aligned}
\left|f_{\beta}(T z+\beta y)-f_{n}\left(T y_{n}\right)\right| & =\left|f_{\beta}(T z+\beta y)-g_{n}\left(T z+\beta y-\beta y+\beta T x_{n}\right)\right| \\
& \leq\left|\left(f_{\beta}-g_{n}\right)(T z+\beta y)\right|+|\beta|\left\|y-T x_{n}\right\| /\left\|y_{n}\right\|,
\end{aligned}
$$

so $f_{\beta}(T z+\beta y)$ is a cluster point of $W(T)$.

Furthermore $f(y)=f_{\beta}(y)$ and $f(T y)=f_{\beta}(T y)$, by the choice of $z$, so

$$
\begin{aligned}
\left|f_{\beta}(T z+B y)-\alpha\right| & =\left|f_{\beta}(T z+B y)-f(T z+\beta y)\right| \\
& =\left|\left(f_{\beta}-f\right)(T z-T y)+\left(f_{\beta}-f\right)(T y+B y)\right| \\
& =\left|\left(f_{\beta}-f\right)(T z-T y)\right|<\varepsilon .
\end{aligned}
$$

Thus a cluster point of $W(T)$ is within $\varepsilon$ of $\alpha$, completing the proof of the theorem.

\section{Reference}

[1] J.R. Giles and G. Joseph, "The numerical ranges of unbounded operators", Bull. Austral. Math. Soc. 11 (1974), 31-36.

Department of Pure Mathematics and Mathematical Statistics, University of Cambridge,

Cambridge,

England. 